\title{
BCI based control of wheelchair for mobility
}

\author{
Pallavi kohli ,Krishna K. Warhade ${ }^{2}$ \\ ${ }^{*}$ M.E. student at Department of Electronics and Telecommunication, MITCOE, Pune, Maharashtra, India \\ ${ }^{\dagger}$ Professor at Department of Electronics and Telecommunication, MITCOE, Pune, Maharashtra, India \\ Email: Pallavi.kohli04@gmail.com, ${ }^{2}$ Krishna.warhade@mitcoe.edu.
}

Abstract-Brain Computer Interface(BCI) is a platform that provides an opportunity to develop an alternative way of communication for patients who lose their ability to communicate. BCI helps people restore their mobility which is driving a wheelchair in this case. With the help of this technique one can understand the intended thoughts of such users. In this paper, different signals from such patients have been analyzed. Essential steps like pre-processing, feature extraction and classification are performed that helps us in analyzing the signal.

Keyword- Brain computer interface, Wheelchair, BCI application

\section{INTRODUCTION}

Brain Computer Interface provides us with a platform that helps us in creating a communication link between the users and the computer systems. It allows us to communicate by using only our brains with no involvement of the actual muscles that are usually involved in the process of communication. The research communities around the world are developing different medical applications based on BCI thus helping patients who have lost their ability to communicate thus providing them mobility[1].

\section{Signal acquisition}

The signal acquisition step involves signal being recorded from the user's brain using any of the acquisition methods which are invasive, partially invasive or non-invasive BCI acquisition technique. Generally the signals taken from the human body are very low in amplitude. For proper analysis of the signal we need to amplify the signal [2].

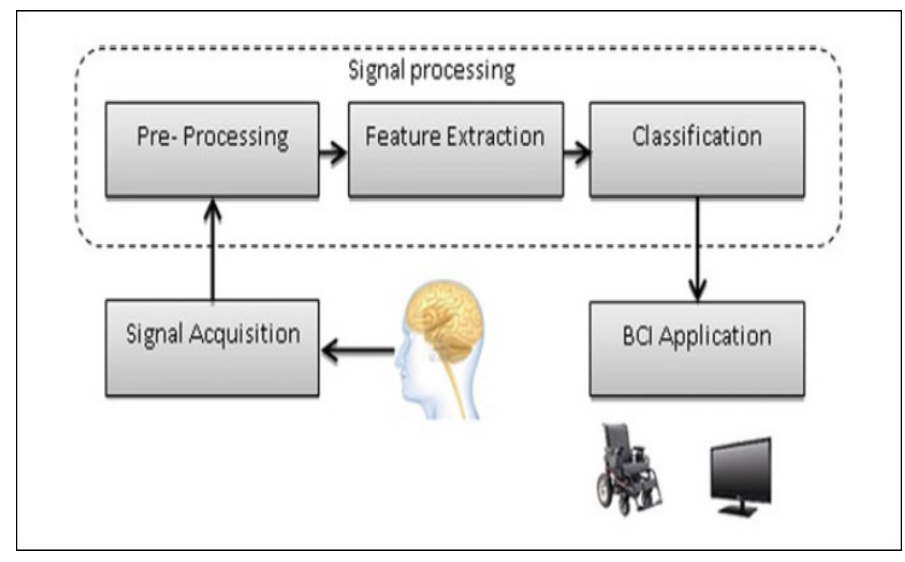

Fig. 1. BCI signal processing[2]

\section{Signal processing}

The signals acquired from the surface of the brain needs to be processed. The steps are discussed below :

Pre-processing

Acquired signal from the brain suffers from a lot of noise. The signal gets interfered by different sources of noise like heavy breathing, coughing or even eye blinking. This noise has to be removed in order to prepare our signal for further processing which makes our signal more efficient.[2].

Feature extraction

The feature extraction in BCI can be done using time analysis, frequency analysis, time-frequency analysis or Power Spectral Distribution(PSD). By using any of the method mentioned above we extract different features from our signal of interest. 


\section{Classification}

Classification basically involves training our classifier to take the accurate decision. In this we identify a specific patterns which are being generated and then translated into commands to perform the intended action desired by the user [13]. Some common and efficient classifiers are linear classifiers, neural networks, nearest neighbor classifier. Linear Discriminant Analysis (LDA) and Support Vector Machine (SVM).

\section{BCI application}

BCI application can be developed once we process the bio-signal, extract the features and classify them using the appropriate classifier which is controlling a wheelchair in our case.

Fig.2 shows the brain computer interface which is classified into three main categories.

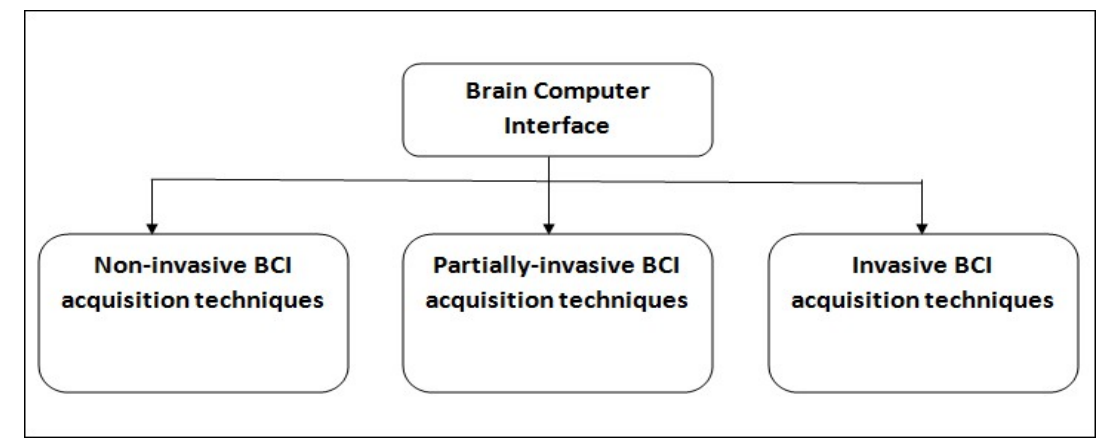

Fig. 2 Types of brain computer interface

(1) Invasive BCI Acquisition Technique

Invasive BCI acquisition is a technique in which brain's electrical activity is measured by placing the electrodes inside the brain. The placement of electrodes is done by performing a critical surgery. The main advantage of this technique is that the signal acquired is of the highest quality as the signal is recorded directly from inside of the brain. This technique has one disadvantage that a critical surgery needs to be performed for placing the electrodes deep inside the brain[2].

(2) Partially Invasive BCI Acquisition Technique

Another technique in which the electrodes are placed inside the skull but over the surface of the brain is partially invasive method[2]. The strength of the signal acquired for this method is weaker than those acquired using the invasive method[3].

\section{(3) Non Invasive BCI Acquisition Technique}

Non invasive technique is a method of recording the brain's electrical activity by placing the electrodes over the scalp. The electrodes are positioned over the scalp in accordance to the international standards. Among the non-invasive methods, EEG(Electroencephalography) is the most reliable and safe method. [3]. The other devices can be fMRI(functional Magnetic Resonance Imaging), MEG( Magnetoencephalography) and fNIRS(Field Near Infrared Spectroscopy).

\section{RELATED WORK}

This section discusses about some of the similar work done in the field of brain computer interface using different acquisition method for recording the brain signal.

A partially invasive signal acquisition method was used for recording the brain signal by Leuthardt et al.[4]. The signals were recorded using Electrocorticography (ECoG). A BCI system was developed which was used to control the cursor movement in 1-D on a computer screen. The cursor was controlled by performing the motor imagery actions. The placement of the electrodes was done over the sensorimotor cortex for about 3-8 days. While the user performed different motor imagery tasks, the respective frequency bands were identified.

Iturrate Iaki, et al.[5] developed a wheelchair whose movement was controlled by using the P300 brain signal. The wheelchair was also coupled with a automated navigation system. When a person sits on a wheelchair, he is provided with the reconstruction of the scenario which is in front of his eyes on a computer screen. P300 signal is elicited whenever the user focuses on the desired location where he wants to drive his wheelchair. Signal processing helps in identifying the desired location and it is sent to automated navigation system. The wheelchair is then driven to the intended target. The wheelchair is also coupled with a laser scanner in order to avoid any kind of obstacle in the path. 
Lal Thomas Navin, et al.[6] proposed an alternative solution to BCI which is EEG based. EEG based BCI has low signal to noise ratio that makes it inefficient. A study was performed by placing electrode grids over motor cortex area of the brain. The subjects chosen for the study were 3 epileptic patients. The subjects performed different imagery tongue and finger movements. Results were recorded for the same.

A classical conditioning hypothesis was proposed for Alzheimer disease by Liberati Giulia, et al.[7]. As we know that people suffering from degenerative disorder start to lose their communication ability. This study can be of great help for such people. By associating the basic thoughts like "yes" with positive stimuli and "no" with negative stimuli one can convey the basic thoughts.

Krishna D. Hari, et al.[8] developed a wheelchair using two different brain signals. One signal was P300 signal and the other signal was generated whenever the person performed the motor imagery action. The performance of the wheelchair was analyzed for different classifiers. The classifier that gave the shortest path was chosen as the best classifier for the wheelchair. An electrode cap consisting of 24 electrodes was placed on the scalp pf the subject. He then performed different imagery movements with a total of 256 trials.

\section{PROPOSED SYSTEM}

A non-invasive BCI acquisition method has been proposed in the Fig. 3 to record the signals from the brain for analysis. The block diagram for the proposed system is shown below.

\section{Data acquisition}

The first step is to record the signals from the brain. For that purpose we are planning to use portable EEG setup to store and analyze the EEG signals from the brain by properly placing the electrodes onto the scalp on the positions FP1 and A1.

\section{Signal filtering}

After we have recorded the signals from the brain we need to remove the unwanted components from the signal. The unwanted signals can be artifact due to eye blinking, heavy breathing or coughing. The filtering can be done by applying low pass filter and notch filter to remove the power line noise.

\section{Feature extraction}

Next we extract the features from the signal that has been recorded. The features can be extracted using Power Spectral Density (PSD), Wavelet transform and energy operator.

\section{Classification}

The next task is to classify the signals and identify the pattern. For signal classification we have different algorithms but because of the accuracy of the Support vector machines(SVMs) classifier, we can choose this classifier for controlling our wheelchair.

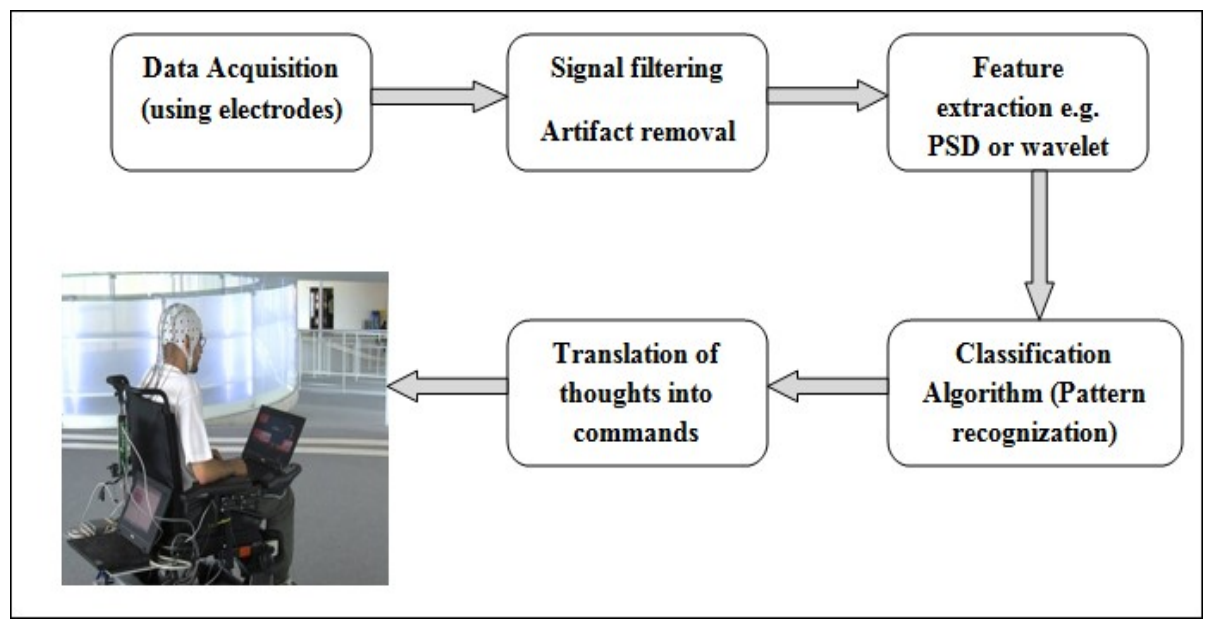

Fig. 3. Proposed system block diagram

\section{Translation into commands}

After the classification has been done, the next step is to simply convert the intended thoughts of the subject into commands that will help in driving the wheelchair in desired direction.

\section{BCI application}

Finally we should be able to perform the desired task and move the wheelchair in the desired direction. 


\section{EXPERIMENTAL RESULTS}

The setup used for capturing the brain signals is shown in Fig. 4 and it mainly consists of instrumentation and operational amplifiers. Single channel electrode is used with two electrodes to be placed over the forehead and reference electrode is placed on the earlobe.

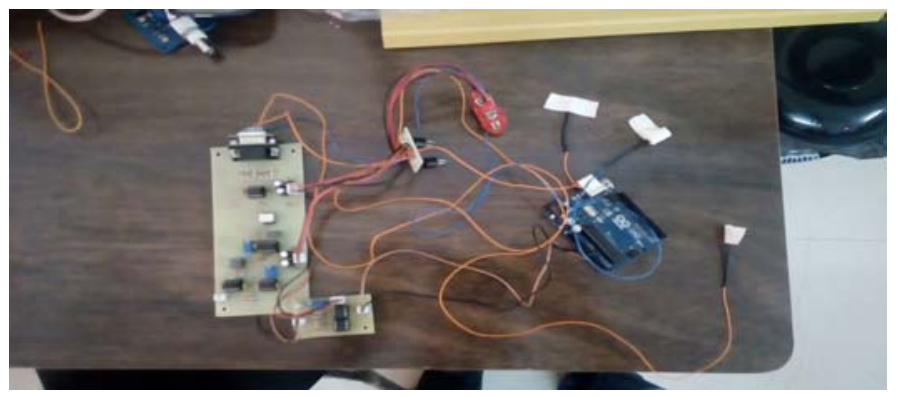

Fig. 4. EEG capturing unit

After the signal has been recorded, it is loaded into the MATLAB and the same is plotted. The next step involves pre-processing of the loaded signal which is corrupted because of artifacts. To remove this noise we use zero-phase low pass filter. The next step involves in pre-processing is to remove the baseline from our signal. The further steps involve to extract the important features from our loaded signal. The main purpose of feature extraction is to extract the important information from the signal of interest. Fig. 5 shows the power spectral density of the loaded signal and Fig. 6 shows the energy of the signal calculated using Teager energy operator (TEO).

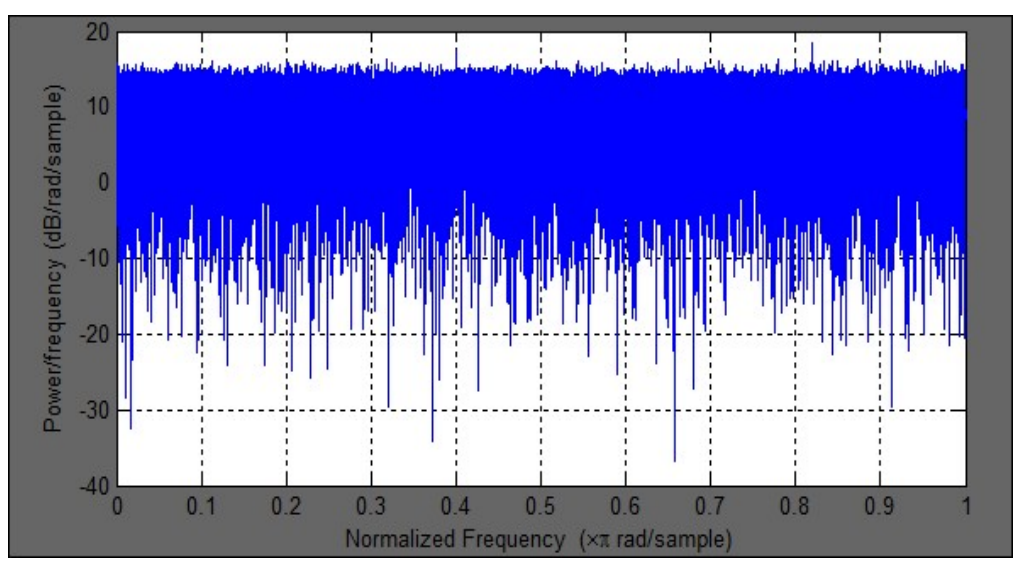

Fig. 5. Power spectral density

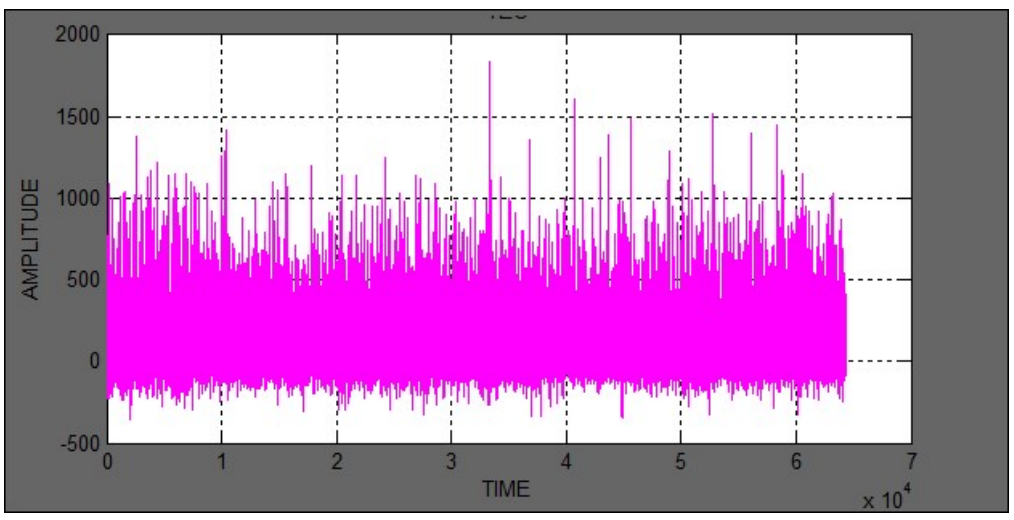

Fig. 6. Energy of the signal 


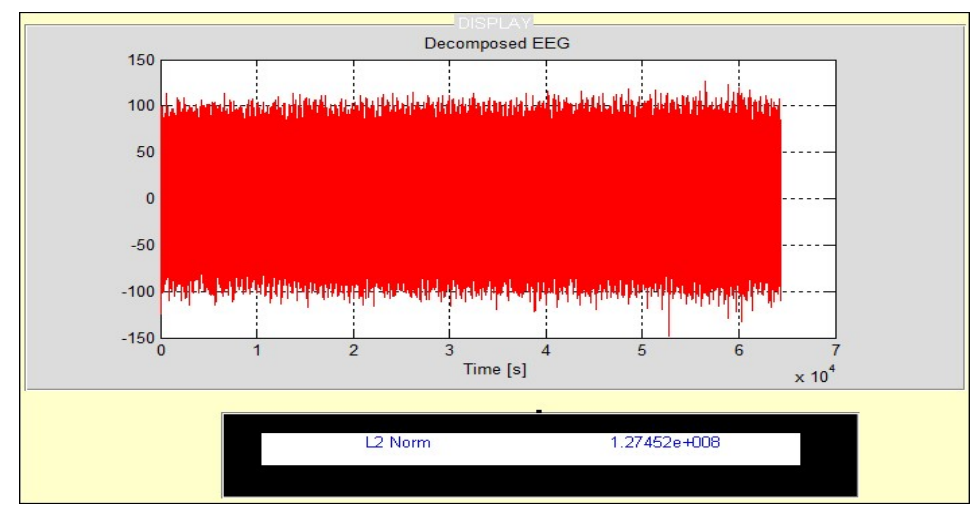

Fig. 7. Energy of decomposed signal

Decomposition of the signal is done using Dabachies wavelet at level 2 and energy is calculated. Fig. 7 shows energy of the decomposed signal.

Decomposition is then performed using Symlet wavelet at level 4. Here we find the detailed as well as the approximate coefficients. Fig. 8 shows scale 1 detailed coefficient.

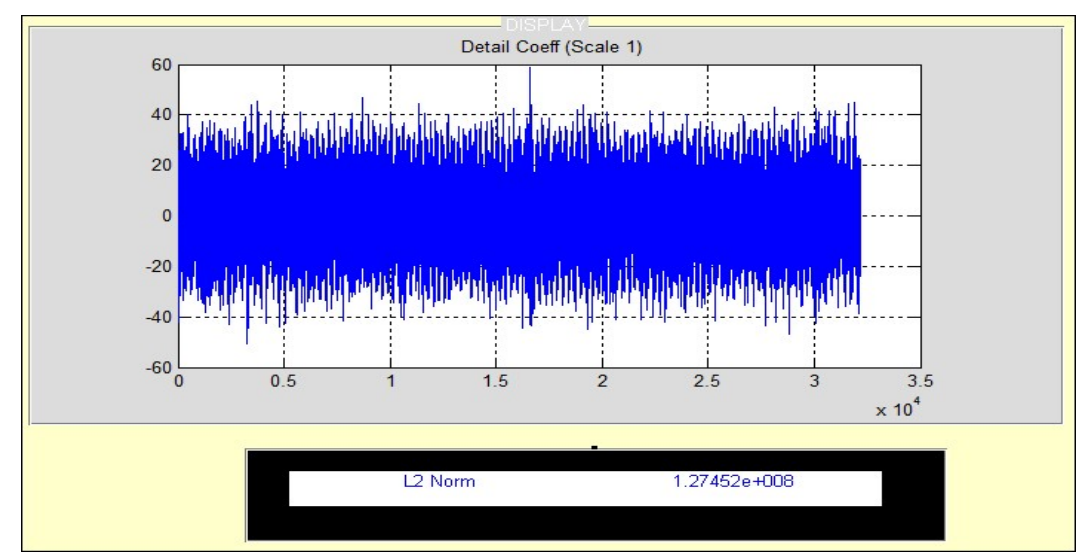

Fig. 8. Scale 1 detailed coefficient

Fig. 9 shows scale 2 detailed coefficient.

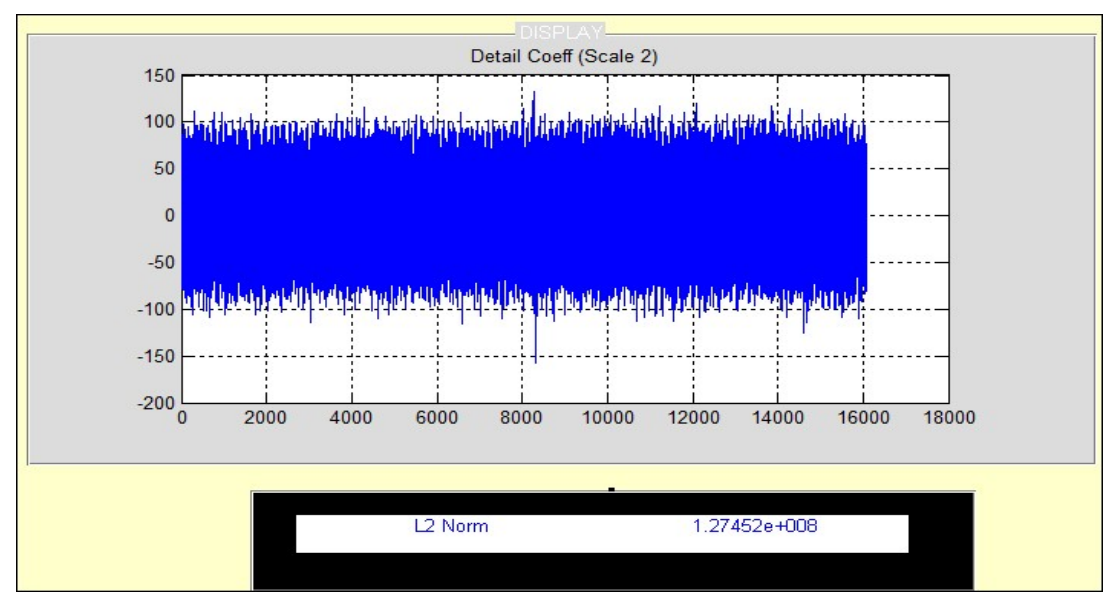

Fig. 9. Scale 2 detailed coefficient 
After we load one of the recorded EEG signal from the available EEG dataset we get the different features. The results are stored in a file. The features can then be analyzed for interpreting the thoughts of the user i.e. to understand the intended direction.

The Table I below shows the values of the different features that were extracted from the signal of interest. These include various statistical features like mean, mode, range, energy, kurtosis, skewness, median, standard deviation and FFT. As the number of extracted features increases, the more will be the accuracy of our classifier.

TABLE I EXTRACTED FEATURE VALUES

\begin{tabular}{|l|l|l|l|l|}
\hline FFT & Energy & Teager Energy & Compressed Energy & Mean \\
\hline $1.53 \mathrm{e}+06$ & $1.83 \mathrm{e}+08$ & $1.19 \mathrm{e}+11$ & $1.27 \mathrm{e}+08$ & -0.088 \\
\hline
\end{tabular}

TABLE II XTRACTED FEATURE VALUES

\begin{tabular}{|l|l|l|l|l|l|}
\hline Kurtosis & Skewness & Median & Mode & $\begin{array}{l}\text { Standard } \\
\text { deviation }\end{array}$ & Range \\
\hline 2.48 & -0.056 & 0.251 & $-1.53 \mathrm{e}+02$ & 28.51 & $2.66 \mathrm{e}+02$ \\
\hline
\end{tabular}

The values of the extracted features which are shown in the above table are compared with the values in training data. The training data consists of the standard values for these 11 features namely FFT, energy, Teager energy, Compressed energy, mean, kurtosis, median, mode, standard deviation and range when the person thinks about a particular direction. These directions include left, right, forward and backward direction. These values are arranged in $11 \times 20$ matrix.

We have 5 training datasets for each direction.

1) 5 training datasets- Right direction

2) 5 training datasets- Left direction

3) 5 training datasets- Forward direction

4) 5 training datasets- Backward direction

After we load a dataset in our GUI, perform pre-processing onto it, extract the above mentioned 11 features, these values are stored in $11 \times 1$ matrix. The minimum distance classifier then compares the extracted values in the $11 \times 1$ matrix with all the values in the training dataset which are in the $11 \times 20$ matrix . The classifier then calculates the distance of extracted values from each value in our training dataset which are arranged in a $1 \times 20$ matrix. The dataset having minimum distance from the extracted values is expected to be the result of the user's thought and the wheelchair can be driven to that particular direction.

By using the training datasets we train our classifier thus making it capable of taking the accurate decision. The Table III shows the normalized difference of the extracted features and the standard values available in the 20 training dataset for all the for all the four directions - right, left, forward and backward. The difference between each dataset and the extracted features is arranged from sequence 1 to 20 in Table III. From the Table given below we can clearly see that the difference given under index number 14 is having the minimum distance from the database which contains the extracted features that we recorded. Thus the classifier gives the decision depending on this minimum distance.

TABLE III DISTANCE CALCULATED BY MINIMUM DISTANCE CLASSIFIER

\begin{tabular}{|c|c|c|c|c|c|c|c|c|c|}
\hline 1. & 2. & 3. & 4. & 5. & 6. & 7. & 8. & 9. & 10. \\
\hline $1.178 \mathrm{e}+11$ & $1.167 \mathrm{e}+11$ & $1.073 \mathrm{e}+11$ & $1.177 \mathrm{e}+11$ & $1.180 \mathrm{e}+11$ & $1.181 \mathrm{e}+11$ & $1.183 \mathrm{e}+11$ & $1.183 \mathrm{e}+11$ & $1.188 \mathrm{e}+11$ & $1.179 \mathrm{e}+11$ \\
\hline
\end{tabular}

\begin{tabular}{|c|c|c|c|c|c|c|c|c|c|}
\hline 11. & 12. & 13. & 14. & 15. & 16. & 17. & 18. & 19. & 20. \\
\hline $9.680 \mathrm{e}+11$ & $9.228 \mathrm{e}+11$ & $1.045 \mathrm{e}+11$ & $1.491 \mathrm{e}-08$ & $1.166 \mathrm{e}+11$ & $1.180 \mathrm{e}+11$ & $1.188 \mathrm{e}+11$ & $1.191 \mathrm{e}+11$ & $1.191 \mathrm{e}+11$ & $\overline{5} .760+11$ \\
\hline
\end{tabular}




\section{CONCLUSION}

The aim is to develop a wheelchair based on BCI. For acquisition of the signal from the brain noninvasive acquisition method is used. Electroencephalography (EEG) is the most commonly used non-invasive method. After the signals are recorded from the brain, processing of the signal is done. The pre-processing steps involve amplification and filtering. The key features from the recorded signal are then extracted using different feature extraction methods like power spectral density, energy, kurtosis, mean, mode, median, range, standard deviation and compressed signal energy. The extracted features are then classified using the minimum distance classifier. The wheelchair then moves in the intended direction on the basis of the result given by the classifier.

\section{REFERENCES}

[1] Abdulkader, Sarah N., Ayman Atia, and Mostafa-Sami M. Mostafa. "Brain computer interfacing: Applications and challenges”,Egyptian Informatics Journal, vol. 16, iss. 2, pp. 213-230, 2015.

[2] Ramadan, Rabie A., S. Refat, Marwa A. Elshahed, and Rasha A. Ali. "Basics of Brain Computer Interface", Springer InternationalPublishing, pp. 31-50. , 2015.

[3] Anupama, H.S., Cauvery, N.K. and Lingaraju, G.M.“ Brain computer interface and its types-a study”, International Journal of Advances in Engineering Technology, vol. 3, iss. 2, p.739, 2012.

[4] Leuthardt, E.C., Schalk, G., Wolpaw, J.R., Ojemann, J.G. and Moran, D.W.,“ A braincomputer interface using electrocorticographic signals in humans”, Journal of neural engineering, vol. 1, iss. 2, p.63, 2004.

[5] Iturrate, I., Antelis, J.M., Kubler, A. and Minguez, J.,“A noninvasive brain-actuated wheelchair based on a P300 neurophysiological protocol and automated navigation”, IEEE Transactions on Robotics, vol. 25, iss. 3, pp.614-627, 2009.

[6] Lal, T.N., Hinterberger, T., Widman, G., Schrder, M., Hill, N.J., Rosenstiel, W., Elger, C.E., Schlkopf, B. and Birbaumer, N., “Methods Towards Invasive Human Brain Computer Interfaces”, NIPS, pp. 737-744, 2004, December.

[7] Liberati, G., Da Rocha, J.L.D., Van Der Heiden, L., Raffone, A., Birbaumer, N., Olivetti Belardinelli, M. and Sitaram, R.,"Toward a brain-computer interface for Alzheimer's disease patients by combining classical conditioning and brain state classification”, Journal of Alzheimer's Disease, vol. 31, iss. s3, pp.S211-S220, 2012.

[8] Krishna, D.H., Sengupta, R., Pasha, I.A. and Savithri, T.S., "Brain Computer Interface For Mobility-A Case Study With ALS Patient”.

[9] Abiyev, R.H., Akkaya, N., Aytac, E., Gnsel, I. and a?man, A.,"Brain-Computer Interface for Control of Wheelchair Using Fuzzy Neural Networks", BioMed research international, 2016.

[10] Leeb,R.,Friedman,D., Mller-Putz, G.R., Scherer, R., Slater, M. and Pfurtscheller, G.,“ Self-paced (asynchronous) BCI control of wheelchair in virtual environments: a case study with a tetraplegic”, Computational intelligence and neuroscience, 2007.

[11] Huang, D., Qian, K., Fei, D.Y., Jia, W., Chen, X. and Bai, O.,“ Electroencephalography (EEG)-based brain computer interface (BCI): A 2-D virtual wheelchair control based on event-related desynchronization/synchronization and state control”, IEEE Transactions on Neural Systems and Rehabilitation Engineering, vol. 20, iss. 3, pp.379-388, 2012

[12] Rebsamen, B., Burdet, E., Guan, C., Zhang, H., Teo, C.L., Zeng, Q., Laugier, C. and Ang Jr, M.H., “Controlling a wheelchair indoors using thought”, IEEE intelligent systems, vol. 22, iss. 2, 2007.

[13] Lotte, F., Congedo, M., Lcuyer, A., Lamarche, F. and Arnaldi, B., "A review of classification algorithms for EEG-based brain computer interfaces”, Journal of neural engineering, vol. 4, iss. 2, 2007.

\section{Author ProfiLe}

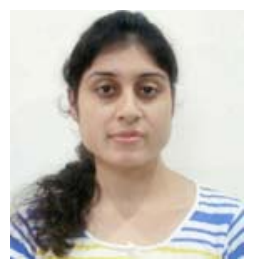

Pallavi Kohli received Bachelor of Technology in Electronics and Communication Engineering in 2013 from Lovely Professional University, Phagwara, India. She is currently pursuing Master of Engineering in VLSI and Embedded Systems from MIT College of Engineering, Pune, India. Her research interest includes signal processing, biomedical and Embedded Systems.

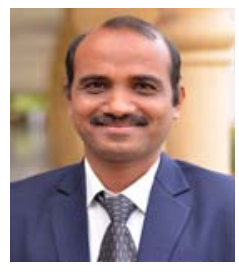

Krishna K. Warhade received the Bachelor of Engineering in Electronics in 1995 and Master of Engineering in Instrumentation in 1999 both from Shri Guru Govind Singhaji Institute of Engineering and Technology, Nanded, India and PhD in Nov 2010 from the department of Electrical Engineering, Indian Institute of Technology, Bombay, India. He has 21 years of teaching experience, he is currently working as a Dean R \& D at MIT College of Engineering, Pune, India. His research interest are in the area of Image Processing, Signal Processing, Video shot boundary detection, Wavelets and biomedical signal processing. 